九州大学学術情報リポジトリ

Kyushu University Institutional Repository

\title{
A MATHEMATICAL FORMULATION OF INFORMATION NETWORK SYSTEM AND ITS IMPLICATIONS TO ARTIFICIAL INTELLIGENCE
}

Kitagawa, Toshio

Director of International Institute for Advanced Study of Social Information Science | Honorary Professor of Kyushu University

https://doi.org/10.5109/13111

出版情報: 統計数理研究. 17 (3/4), pp. 23-38，1977-03. Research Association of Statistical Sciences バージョン：

権利関係 : 


\title{
A MATHEMATICAL FORMULATION OF INFORMATION NETWORK SYSTEM AND ITS IMPLICATIONS TO ARTIFICIAL INTELLIGENCE*
}

\author{
By \\ Tosio KitagaWA** \\ (Received April 1, 1976)
}

\section{Introdution}

The purpose of the present paper is to give a general outline of our research attitudes concerning three types of information processing network systems, namely,

(N) neural network system

(C) computer network system

(S) social information network system,

with particular emphasis to the following two topics (a) and( b).

The topics (a) covers the problems and the methods which we should consider in a mathematical formulation of $(\mathrm{N})$. The topics (b) is concerned with an application of the methodology obtained in $(\mathrm{N})$ to $(\mathrm{S})$. There are two crucial features (i) and (ii) of our research attitudes: (i) it is our views that (C) will be better discussed after some achievements will have been established with reference to $(N)$ and (S); (ii) we believe that artificial intelligence will be better discussed after some deep observations on the characteristic features of integrated and intelligent information systems will have been obtained.

These two points (i) and (ii) do not seem to us to be authentic in the present current research attitudes. In fact there are a lot of technically advanced approaches to $(\mathrm{C})$ on the basis of computer science and technology achievements, which are obviously indispensable to the establishment of (C). But the rôles and the functions of $(C)$ can be neither fully described nor definitely formulated without entering into discussions of the crucial features to be expected for integrated and intelligent information processing system, which is closely related to artificial intelligence. Artificial intelligence is to be discussed in connection with (C).

* The work is done as a member in the research group "C.2": The structure of data base and theory of information retrieval belonging to the Special Research Project "Advanced In formation Processing of Large Scale Data over a Broad Area". The paper was presented to the Third International Congress of Cybernetics and Systems held in Bucharest, Romania, August, 25th-29th, 1975.

** Honorary Professor of Kyushu University, Director of International Institute for Advanced Study of Social Information Science. 
Our emphasis is to investigate artificial intelligence not confining to the purpose of inventing mechanical imitation manipulating in place of human sensory organs but rather concentrating our efforts to cognitive functions of human beings regarding deduction, induction and abduction, as we have emphasised in our papers [3], [4], [8], [9], [10], [12]. For these points of view there are two indispensable research directions. The first direction is to have accurate and detailed observations on the neural dynamics of brain activities. These observations can be obtained for our present purpose not so much through direct investigations on physiological activities of brain and nervous systems but through mathematical analysis of dynamical behaviours of solution of neural equations. The second direction is oriented to social functions of information systems, through which human beings can have attained at both the present stage of civilization and the present capacities of brain activities. In view of these fundamental facts we should prefer to enter into the investigation of $(\mathrm{S})$, with some adequate mathematical formulation.

In Section 2 we shall discuss two mathematical formulations of brain activities due to Wiener [21] and Caianiello [1] and we shall make some critical observations upon the usages and the limitations of these two approaches with reference to the candidate topics such as

$\left(1^{\circ}\right)$ forms of inner connectivity construction,

$\left(2^{\circ}\right)$ functions of inner connectivity construction,

$\left(3^{\circ}\right)$ outside input giving regulation of brain activities,

$\left(4^{\circ}\right)$ learning processes in brain activities.

In Section 3 we shall discuss each of these four topics in each of four subsections in such a way that each of the topics $\left(1^{\circ}\right),\left(2^{\circ}\right),\left(3^{\circ}\right)$ and $\left(4^{\circ}\right)$ will be allocated to Subsections $3.1,3.2,3.3$ and 3.4 respectively. Section 4 is devoted to an evaluation scheme of information system given by the general standpoint of informative logics with reference to three subspaces called (I) control, (II) eizon and (III) creation.

The purpose of Section 5 is to give a mathematical model of social information system and to point out some crucial problems which should be solved in this connection.

\section{System of neural equations}

There are many mathematical formulations which will aim to describe brain activities, but we shall choose two characteristic formulations for our present purpose. The first one is due to Wiener [21] who gave nonlinear random theory which has a deep connection with neural activities. The second one is due to Caianiello [1] who gave a systematic approach to brain activities by introducing a system of neural equations consisting of

(I) neuronic (or decision) equation (NE),

(II) mnemonic (or evolution) equations (ME), and

(III) adiabatic learning hypothesis (or rule) (ALH).

We are not going to enter into detailed comparisons between these two char- 
acteristic approaches apart from the following global observations. The mathematical formulation due to Wiener [21] aims simply to provide us with sample functions drawn from some stationary stochastic process and to introduce correlation analysis of the non-linear functionals of these sample functions. On the other hand the Caianiello equation system definitely assumes $N$ neurons each of which has two exclusive states (i) firing and (ii) non-firing, which will be denoted by 1 and 0 respectively.

Let the state of the $i$-th neuron at the time point $t$ be denoted by $X_{i}(t)$ (i= $1,2, \cdots, N)$. Then the system of $N$ neural equations with time lag $(n-1)$ is given by

$$
\boldsymbol{X}(t+\tau)=\mathbf{1}[A X(t)-\Theta(\cdot)]
$$

where



$$
\begin{aligned}
& A \equiv\left(a_{i j}(k) ; i, j=1,2, \cdots, N: k=0,1,2, \cdots, n-1\right)
\end{aligned}
$$

and

$$
\Theta(t) \risingdotseq\left(\theta_{1}(t), \theta_{2}(t), \cdots, \theta_{N}(t)\right),
$$

with the vector threshold function $\mathbf{1}[u]$ for $u=\left(u_{1}, u_{2}, \cdots, u_{N}\right)$ which is defined by

$$
\mathbf{1}: u=\left(u_{1}, u_{2}, \cdots, u_{N}\right) \rightarrow\left(1\left[u_{1}\right], 1\left[u_{2}\right], \cdots, 1\left[u_{N}\right]\right),
$$

where for real number $u_{j}(j=1,2, \cdots, N)$

$$
1\left[u_{j}\right]= \begin{cases}0 & \text { for } u_{j} \leqq 0 \\ 1 & \text { for } u_{j}>0\end{cases}
$$

See also Caianiello [1] and Kitagawa [6], [11], [13].

The points which we want to emphasise here with respect to $(2.01)$ are the following items (a) and (b).

(a) In spite of the assumption that the matrix $A=\left(a_{i j}(k)\right)$ should be definitely determined, there are no needs for precise determination of each of coefficients, because there are a great deal of tolerances among the values of the coefficients within which the system of the equations can work precisely in the same way. In fact it would be too severe to require a precise determination of the coefficients, when at least either of the two structural sizes regarding networks, namely, $N$ and $n$ becomes fairly large.

(b) In spite of the assertion given in (a), there must be a firm ground in order that we may assume randomness of the coefficients themselves even in the case of large $N$ and/or large $n$.

With these two assertions in mind, we may consider that the main feature of the 
mathematical formulation due to Caianiello [1] lies in giving deterministic transition mechanism in sharp contrast with the purely stochastic theory due to Wiener [21].

The comparison between these two formulations leads us to an understanding of information structure by locating these two approaches in a certain coordinate system, which we coin as the first coordinate system (I) in the three coordinate systems in the logics of information science in our paper Kitagawa [3], [4], [8], [9], [10], [12], that is, in the informative logics in terms of our recent paper Kitagawa [12]. The objectivity aspects in describing the structure of information are concerned with $\left(a_{1}\right)$ pattern $\left(a_{2}\right)$ chaos and $\left(a_{3}\right)$ transformation, as we have explained in detail in these papers of ours. In view of the general framework it can be pointed out that Caianiello [1] refers to $\left(a_{1}\right)$ and $\left(a_{3}\right)$ while Wiener [21] is chiefly concerned with $\left(a_{2}\right)$ and $\left(a_{3}\right)$. This observation suggests us that there should be some amalgamation of these two approaches by which we may expect to have a deeper insight into the dynamical behaviours associated with brain activities. Having such a general idea of amalgamation in mind, we shall start with the mathematical formulation of what Caianiello [1] calls neuronic (or decision) equation (NE) rather than with a mathematical scheme equipped with more complicated formulas, and we shall seek for its elaboration in view of information functions which should be expected for our formulation. There are three aspects of information functions, $(\alpha)$ cognition, $(\beta)$ direction, and $(\gamma)$ evaluation, as we have explaiued in our papers [3], [4], [8], [9], [10], [12]. With reference to information processing procedures, these information functions can be observed in each of the following five processes: [1] information processing process of receiving input, [2] information processing regarding acquisition, reduction, and evaluation, [3] information processing as structuring of information, [4] information storage and retrieval, [5] information circulations and usages. As we have explained in our paper [8], these information processing processes are connected with the notion of brainware associated with integrated and intelligent information system.

\section{Neural equation approaches}

In this Section 3 we shall give several observations on the various rôles of neural equation approaches. Subsection 3.1 is concerned with the rôles and the implications of the inner connectivity coefficient matrix $A$. Subsection 3.2 is devoted to a discussion of the five information processing processes from the standpoint of neural equation dynamics. In Subsection 3.3 we turn to a discussion regarding the rôles of input function $\Theta(t)$ which is being assigned from outside to our network system. In the consequence of these discussions it will be shown that there is a necessity to introduce learning processes to our network system formulation, as we shall discuss in Subsection 3.4.

\section{1. Morphological observations on the inner connectivity cofficient matrix $A$}

The purpose of this Section 3.1 is to give various possibilities of introducing some sort of structuring into the inner connectivity coefficient matrix $A$, as shown 
in the following five items.

(i) Connectedness and separability

In the inner connectivity matrix $A$ it may happen that some coefficients may have larger absolute values than others, leading, in the consequence, groupings of some neurons in which neurons in the same group are strongly mutually connected and neurons in different group are losely connected if not. Connectedness within group and separability between group come together. It is the merit of the inner connectivity matrix $A$ that it will admit these fundamental features by specifying to some extent the relative magnitudes of some inner connectivity coefficients in A. (See Kitagawa [11].)

(ii) Hierachies by multistage stratifications and subnetworks

The integration aspects in system formation, which are crucial in any information science approaches as we have shown in our paper [12], can be also found in neural equation dynamics of brain activities through adequate choice of the inner connectivity coefficients. In particular the principle of construction including sequential arrays and parallel ones and the principle of integration including black box method and feedback method can be realized in neural equation dynamics of brain activities by bringing various sorts of multistage stratifications and subnet schemes into the construction of the inner connectivity coefficient matrix $A$. In these ways the multitudes of inputs can be reflected into multitudes of outputs by means of variability of transformations being involved to introduce while the inputs are being processed along the inner connectivity mechanism defined by the matrix $A$ in which multistage stratifications and subnetwork consisting of neurons are being introduced.

(iii) Hierachical structuring induced by stratified response times

It seems to us to be crucially important to introduce a set of different time units $\left\{\tau_{h}\right\}(h=1,2, \cdots, q)$ in order to make a distinction among brain phenomena of different duration times. Instead of the system of neural equations (2.01) which has only one time unit $\tau$, our proposal to generalization of $(2.01)$ is based upon a system of neural equations having the following form:

$$
\begin{aligned}
& X_{i}\left(t+\sum_{k=1}^{q} m_{k} \tau_{k}\right) \\
& =1\left[\sum_{k q=0}^{m q-1} \sum_{k q-1=0}^{m_{q-1}-1} \cdots \sum_{k 1=0}^{m_{1}-1} \sum_{j=1}^{N} a_{i j}\left(k_{1}, k_{2}, \cdots, k_{q}\right)\right. \\
& \left.X_{j}\left(t+\sum_{k=1}^{q} k_{k} \tau_{k}\right)-\Theta\left(t+\sum_{k=1}^{q}\left(m_{k}-1\right) \tau_{k}\right)\right], \\
& \quad(i=1,2, \cdots, N)
\end{aligned}
$$

for every set of $q$ non-negative integers $\left(m_{1}, m_{2}, \cdots, m_{q}\right)$.

We are here content with merely proposing such a system of neural equations. It deserves to be discussed throughly in a systematic way. It can be readily observed however that the existence of different time units will involve a set of various reverberation cycles with different duration times, as can be expected from the results already being established by many authors with respect to the system 
of neural equations. It also ought to be noted that, so far as neural equation approaches to brain activities are concerned, the existence of a set of different time units may induce a hierachical system of phenomena under certain situations. The details of our considerations will be given in another occasion.

(iv) Structural stability and stochastic stability

Since each inner connectivity coefficient of the matrix $A$ can be subject to some change without involving any difference of transition behaviours of the solutions of the system (2.01), it is quite natural, at least from the viewpoint of mathematical treatment, to consider the problem of stochastic stability, in which the matrix $A$ is considered to be a stochastic matrix consisting of stochastic variable $\left\{a_{i j}(k)\right\}$ and we may be concerned with the problem to find out the conditions under which the dynamic behaviours of the system (2.01) remain invariant. There are, however, other problems of stability in which we are concerned with such an invariance of dynamical behaviours of the system when the deterministic matrix $A$ may change within certain domain in the $n N$ enclidian space. This is the problem to be discussed in the general framework of topology, under the name of structural stability in somewhat more generalized sense than those treated by Thom [19] [20].

\subsection{Information processing functions to be expected by neural network systems}

The purpose of this subsection is to show how far information processing functions ennunciated Kitagawa [8] can be realized within a general framework of neural equation systems.

[1] Information processing process of receiving input

The attitude to appeal to neural equation systems is derived from the premises to the effect that these should have biological foundations as discussed ay Lenneberg [14], for the whole information processing scheme to be observed in animals and in human beings. In this connection the whole domain of mathematical apparata provided by the systems of neural equations do seem to us to be worthwhile to be investigated regarding whether and how they are broad enough to cover the whole information processing scheme. Of course there should be a certain obvious limitation of validity of our assertion, because there exist biological existences which have no nervous system at all. But so far as an organism is provided with nervous system, it should be worthwhile to find out a mathematical model by which to describe the procedures how to receive input information from outside world. There have been given various apparata aiming to provide artificial intelligence which imitates some sensory functions. We belive it to be worth while to check the software aspects of these apparata so that we can inquire whether and how far their software aspects are being connected with neural equation approaches.

[2] Information processing regarding acquisition, recuction, and evaluation of input information

The logical implications of these information transformations may sometimes have very deep implication, because they can be only done through pattern recognition in a broad sense. Here we are concerned with rather simplified pictures of acquisitions, reductions and evaluations of input information. Broadly speaking, 
there are two fundamental aspects, i.e., logical operations and arithmetic ones. The logical operations such as 'and', 'or', 'not' and so on can be implied by linear threshold functions which are nothing but specific forms of general neural network formulation given by (2.01). The fundamental arithmetic operations can be considered from the two processes called poolings of information and process of evaluating the information in question. In view of raw input information with which we are now concerned, it is quite natural to consider these information processing procedures in an analogous way to the methodology of statistical quality control. In principle there are two fundamental arithmetic operation processes summation process and differentiation process. It is the essential function of process that each individual stimulus, which is not strong enough to lead us any operation process, can be accumulated so as to attain at some significant conclusion which would result in any application of operation procedure. On the other hand, differentiation process is concerned with comparison of different informations whether ( $i$ ) they should be considered essentially different or (ii) their difference is superficial so that they can and they should be pooled up. The comparison leads to testing of null hypothesis, as well known in statistical theory of testing hypothesis.

[3] Information processing as structuring of information

Here we understand by structuring of information the information processing process in which either one or some combination of (i) pattern recognition, (ii) semantic understanding and (iii) mathematical formulation is being required to obtain. With respect to these profound sophiscated problems, what we want to point out here is that there is a certain set of common features of these three (i), (ii) and (iii) in the sense that we can set up the neural equation approaches which may serve to connect these three different problems within one common framework. Let us explain our viewpoints with regard to this.

Regarding (i) pattern recognition problem, various approaches are substantially concerned with the common set-up which is connected with special cell configurations in cell space, as can be observed from the perception theory by Codd [2], Minsky-Papert [16] and our approach in cell space summarized in Kitagawa [5].

Regarding (ii), semantic analysis of languages, a recent paper of Kitagawa [13] is devoted to show that the reverberation cycle dynamics may be one of the hopeful candidate approaches to interpret the central constituents of the sentence, namely, certain fundamental verbs and nouns. These reverberation cycle dynamics are observed in dynamical phenomena of neural network system.

Although our proposal is still very vague in some respects, the main points which we want to emphasize is rather clear to the effect that a lot of possibilities having deep implications to language phenomena should be systematically explored by investigating reverberation dynamics on the basis of neural equation approaches, as we have shown in our recent paper [13].

[4] Information storage and retrieval

The technical aspects of information storage and retrieval have been fully investigated in documentation and library science, and computer usages for these 
purposes are also being in the stage of rapid developments. In our framework of conceptional arrangements these technical aspects belong to $(\mathrm{C})$ already enunciated in the beginning of Introduction. From the standpoint based upon $(N)$, the phenomena of information storage and retrieval can be described with respect to reverberation cycles to be obtained in neural equation dynamics, since reverberation cycles have been recognized to be the function of memories in brain activities. The correspondence among reverberation cycles and memories can be more fully traced and investigated by means of reverberation cycle dynamics to be observed in solution of neural equations. As we have remarked with regard to (3.01), it is possible to have a hierachical system of reverberation cycles with different time durations which may be considered to correspond to information retrieval in a broad sense. It is noted in this connection that the real function of information retrieval should be understood, from a broad viewpoint of information science and linquistics, as learning and adaptive processes based upon question-answer schemes.

It is the view of the present author that, in spite of the current approaches to information storage and retrieval by computer scientists, in which computer languages along the line of LISP and its ascendants play the dominant roles, we would rather return back to the brain model in which the various aspects of phenomena of memory can be traced and can be investigated. For instance, information storage in our brain can not be identified with the memory function in the computers of the present time. Storages of information in themselves have their history including genesis, growth, break down and disintegration, that is to say, the real pictures of information storage should be self-organizing in the sense of cybernetics. In view of the deep implications of information storage, both the theoretical and the practical problems regarding information retrieval should be recognized with reference to the dynamical behaviours of brain activities shown through the mathematical formulations which we have explained.

[5] Information usages and circulations

This is the research area which has been discussed in social sciences including sociology, mass communication theories, library sciences and documentation, but not in connection with biological foundations including brain activities. Nevertheless it ought to be pointed out that, without feedback connection with information usages and circulations, the four items [1] input information [2] transformation of information [3] structuring information and [4] information storage and retrieval will not be discussed in their full content. This is the reason why we should appeal to the second coordinate system regarding the information function consisting three axises: $(\alpha)$ cognition, $(\beta)$ direction and $(\gamma)$ evaluation which we have introduced in Kitagawa [3], [4]. It will be required to have detailed observations on on human mental activities from both physiological and psychological points of views with an intension to seek for an adequate set of functionals of output functions generated as the results of brain activities on the basis of the neural equation approach. This is certainly an uncultivated area for theoretical studies of brain dynamics. 


\section{3. Regulation by input functions}

There are two fundamental aspects (a) and (b) of regulations which are caused by input functions $\Theta(t)$ in brain dynamics given by the system of neural equations (2.01):

Case (a) A certain input function $\Theta(t)$ induces a certain type of regulation irrespective of the inner connectivity coefficient matrix $A$.

Case (b) A certain input function $\Theta(t)$ induces a certain type of regulation with combination of certain inner connectivity coefficient matrix $A$.

Let us explain these two cases by the following examples.

EXAMPLE 3.1. A simple example of the case (a) is given by a single equation

$$
X(t+1)=1\left[\sum_{k=0}^{n-1} a_{k} X(t-k)-\Theta(t)\right] .
$$

Since $X(t)$ can be either 1 or 0 , the whole set of all the possible states $Q$ will be denoted by

$$
Q=\left\{\delta=\left(\delta_{0}, \delta_{1}, \delta_{2}, \cdots \delta_{n-1}\right) \mid \text { each } \delta_{i}=0 \text { or } 1\right\}
$$

where $\delta_{i}$ corresponds to $X(t-i)(i=0,1,2, \cdots, n-1)$.

For each assigned $\delta$ and any assigned value of $\Theta(t)$ there are two possibilities for value of $X(t+1)$ so that the two possible transitions of $\delta$ by

and

$$
L \delta=\left(\begin{array}{c}
1 \\
\delta_{0} \\
\delta_{1} \\
\vdots \\
\delta_{n-2}
\end{array}\right) \equiv \sigma \delta, \text { say, }
$$

$$
L \delta=\left(\begin{array}{c}
0 \\
\delta_{0} \\
\delta_{1} \\
\vdots \\
\delta_{n-2}
\end{array}\right) \equiv \tau \delta, \text { say. }
$$

It can be readily seen that, when the value of $\Theta(t)$ is positive and very large, $\sigma \delta$ will occur for every $\delta$ irrespective of the inner connectivity coefficients $\boldsymbol{a}=$ $\left(a_{0}, a_{1}, a_{2}, a_{3}, \cdots, a_{n-1}\right)$. Similarly $\tau \delta$ will occur for every $\delta$ irrespective of the inner connectivity coefficients $\boldsymbol{a}=\left(a_{0}, a_{1}, \cdots, a_{n-1}\right)$ when the value of $\Theta(t)$ is negative and very large in its absolute value. This is the situation under which our system is subject to the command of outside world.

EXAMPLE 3.2. In view of the results obtained in a series of our papers, Kitagawa [6], [11], [13], there happens the case (b). For instance we have discussed the case when we have

$$
L \delta=\left(\begin{array}{l}
\overline{\delta_{n-1}} \\
\delta_{0} \\
\delta_{1} \\
\vdots \\
\vdots \\
\delta_{n-2}
\end{array}\right)
$$


under the name of the operator representation $L_{\bar{\beta}(n-1)}$ in our paper [11]. In this case $\sigma \delta$, which is denoted by $L_{\bar{\beta}(0)}$ in our previous paper [6], and $\tau \delta$ will happen respectively according to the condition $\delta_{n-1}=0$ and $\delta_{n-1}=1$.

But as we have investigated in detail in our papers [6], [11], the operator $L_{\bar{\beta}(n-1)}$ can be realized under the specific combinations of conditions on the inner connectivity coefficient vector $\boldsymbol{a}$ and those on the behaviour of the input from outside.

There are two remarks $(\alpha)$ and $(\beta)$ which we want to give in what follows in order to understand the implications of the two cases (a) and (b).

$(\alpha)$ Hierachical constructions both in the inner connectivity coefficient matrix and in the input functions coming from outside can produce a certain set of situations where both of the two cases (a) and (b) can occur in their respective subnet system which may be either global or local. This remark is essential to the learning processes to be discussed in the Subsection 3.4.

$(\beta)$ The crucial aspects of regulation by input function from outside can be observed in the phenomena called assimilation and accomodation in the terminology of J. Piaget [17], [18]. According to J. Piaget [17], [18], assimilation is used to describe the action of organism on surrounding objects, so far as this action depends upon previous behaviours involving the same or similar objecst; and accomodation is used by him to denote the action of environment on the organism, which leads to a simple modifications of the action of the organism. J. Piaget [17], [18] defines an adaptation as an equilibrium between assimilation and accomodation.

\section{4. Learning Process}

In our previous paper [7] we have introduced the notion of biological intelligence and we have explained the relationship between the logic of information science and the general framework of genetic epistemology due to the famous work of $J$. Piaget [17], [18]. According to him, "intelligence constitutes the state of equilibrium towards which tend all the successive adaptations of sensory-motor and cognitive nature as well as the assimilatory and accomodatory interactions between the organism and the environment". (See J. Piaget [17] p. 11) The mechanism of transition proposed in the Piaget theory is an equibration process, which we understand as the most crucial feature of learning as well.

With reference to the neural equation model of brain activities, learning process should be understood as being realized by the change of the inner connectivity coefficient matrix $A$. Our working hypothesis in this connection is the following simple one:

HyPOTHESIS OF CONDITIONING: A repeated application of a certain input function from outside to the neural eqnation system (2.01) will induce a change of the inner connectivity matrix $A$ in the following conditioning way:

$\left(1^{\circ}\right)$ A certain type of reverberation cycles will take place by repeated applications of relativily strong input function from outside, irrespective of the inner connectivity coefficient matrix.

$\left(2^{\circ}\right)$ A duration of the existence of the reverberation cycles induced in $\left(1^{\circ}\right)$ over certain time range will lead to a change of the inner connectivity matrix A. This pro- 
perty of $A$ is called the plasticity of $A$.

$\left(3^{\circ}\right)$ The change of the inner connectivity matrix $A$ enunciated in $\left(2^{\circ}\right)$ amounts to the change of the coefficients $\left\{a_{i j}(k)\right\}$ in such a way that the reverberation cycles in $\left(1^{\circ}\right)$ (and hence in $\left(2^{\circ}\right)$ also) will be conditioned to occur, as will be illustrated in $\left(4^{\circ}\right)$.

$\left(4^{\circ}\right)$ We are not going to introduce any elaborated mathematical formulation to describe the change of the inner connectivity coefficient matrix A. Instead we are proposing an extremely simple and universal procedure of conditioning in the following way.

$A$ repeated application of a certain input function $\Theta(t)$ will induce a reverberation cycle $R$ consisting of states $\left\{s_{k} \mid k=1,2, \cdots, m\right\}$ such that there arises a sequence of transitions $s_{1} \rightarrow s_{2} \rightarrow \cdots \rightarrow s_{k} \rightarrow s_{k+1} \rightarrow s_{m-1} \rightarrow s_{m} \rightarrow s_{1}$. At each state $s_{i}$ there are $2^{N}$ possible designations of transition on the unit time $\left\{s_{i}^{(j)}\right\}\left(j=1,2, \cdots, 2^{N}\right)$ and let us give a numbering such that $s_{i+1}=s_{i}^{(1)}(i=1,2, \cdots, m-1)$ with $s_{m+1}^{(1)}=s_{1}$. The existence of the reverberation cycle $R$ implies that for $i=1,2, \cdots, m$

$$
A s_{i}^{(1)}>A s_{i}^{(j)}\left(j=2,3, \cdots, 2^{N}\right),
$$

that is to say, the system of linear inequalities does hold true, for $i=1,2, \cdots, m$

$$
A\left(s_{i}^{(1)}-s_{i}^{(j)}\right)>0\left(j=2,3, \cdots, 2^{N}\right) .
$$

The essential point of our hypothesis is that the validity of (3.08) for certain time duration will induce the consequence to the effect that the tolerance region of $A$ should be set up so as to be subject to the condition such that (3.08) should hold true for the assigned set of the states $\left\{s_{k}\right\}$.

We are not going to discuss any mechanism by which this will be realized. To find out or to set up such a mechaism is naturally quite important, and should deserve a deep and systematic investigation in itself. Nevertheless what is primarily important in the hypothesis of conditioning is the consequence that the tolerance region for $A$ is now introduced and the actual $A$ should be determined so as to satisfy the condition that it shuold belong to the tolerance region just mentioned. At this stage the tolerance region of $A$ is fairly large enough to admit of a lot of indetermination of the inner connectivity coefficients. A tolerance region of $A$ may specify some inner connectivity coefficients of $\left\{a_{k}\right\}$ so as to define some regions in the $n N$ dimensional space with no specification of other inner connectivity coefficients. But in spite of partial determination of some inner connectivity coefficients and the indetermination of the remaining inner connectivity coefficients, a tolerance region for $A$ will impose in the first place the conditions on $A$ which makes it possible to realize the reverberation cycles under the influence of more generalized input functions which may be different from the original input functions by which $A$ was conditioned so as to remain to this tolerance region of $A$. Secondly, so far as $A$ belongs to the tolerance region of $A$, there may be the possibility to have reverberation cycles different from the set of the original reverberation cycles. It is our proposal that this is the generalization procedure that should be considered as informative function of brain activities. 


\section{Evaluation scheme of information system}

One of three coordinate axises of the second coordinate system, which refers to the function aspects of informations, is concerned with evaluation which consists of three aspects, called as $\left(\gamma_{1}\right)$ control, $\left(\gamma_{2}\right)$ eizon, and $\left(\gamma_{3}\right)$ creation, as we have discussed in a series of our papers [3], [4], [9], [12]. It is perhaps one of the characteristic standpoints of the present author that evaluations scheme of information system should be referred to these three aspects. We are now going to give some remarks which aim to illustrate the reason why this assertion of ours should be clearly enunciated in the present case of evaluation scheme of inforamation network systems.

$\left(\gamma_{1}\right)$ Control aspect in evaluation of information system

As we have analysed in our previous paper [3], [4], [9] evaluation in control subspace is concerned with efficiency aspect based upon the structural aspects of control subspace including $\left(a_{1}\right)$ pattern $\left(b_{1}\right)$ operation and $\left(c_{1}\right)$ optimization which are the basis of observability, controllability and understandability of the system in our concern.

$\left(\gamma_{2}\right)$ Eizon aspect in evaluation scheme of information system

As we have explained in our previous paper [3], [4], [12] evaluation in eizon subspace is concerned with reliability aspect. Mathematical formulations for evaluation scheme in eizon subspace are given by current reliability analysis, redundancy approach, and probabilistic logics and so on. With special reference to information systems which are being established by human design, we should introduce environmentalogical considerations on such information systems. In fact we should consider such information systems to be architectures introduced to information ecospheres which have been partly generated in human societies by some sort of natural evolutionary processes as well as by human designs.

$\left(\gamma_{3}\right)$ Creation aspect in evaluation scheme of information system

As we have explained in our previous papers [3], [4], [9], [10], [12], evaluation scheme in creation subspace is concerned with plasticity aspect.

This assertion implies the possibility of learning process, as we have explained in Subsection 3.3. The creation aspect in evaluation scheme of information system is in fact indispensable, because the formation process of information system should be a learning process requiring architecthnical works of the designers which are based upon their creative powers.

\section{A mathematical model of social information network system and allied problems}

$\left(1^{\circ}\right)$ Let us assume that there is a set $\boldsymbol{X}$ of $N$ nodes whose elements are denoted by $X_{j}(j=1,2, \cdots, N)$ such that

$$
\boldsymbol{X}=\left\{X_{j} ; j=1,2, \cdots, N\right\} .
$$

$\left(2^{\circ}\right)$ A chord $\sigma_{j, k}$ between any two assigned elements $X_{j}$ and $X_{k}$ is defined with 
the reservation that it may be sometimes null $\phi$, when no chord is substantially existent. We shall consider

$$
\Sigma=\left\{\sigma_{j, k} ; j, k=1,2, \cdots, N\right\} .
$$

$\left(3^{\circ}\right)$ Let us assume that to each node $X_{i}$ there exist three types of vector-valued function of time $t$ :

(a) State function of the node $X_{i}$ at time $t$ which is denoted by $X_{i}(t) \quad(i=$ $1,2, \cdots, N)$.

(b) Output function from the node $X_{i}$ to the node $X_{j}$ at time $t$ which is denoted by $O_{i j}(t) \quad(i, j=1,2, \cdots, N)$.

(c) Input function to the node $X_{i}$ from the node $X_{k}$ at time $t$, which is denoted by $e_{k i}(t)(i, k=1,2, \cdots, N)$. The input function to the node $X_{i}$ at time $t$ is defined by the vector sum

$$
e_{i}(t)=\sum_{k=1}^{N} e_{k i}(t)
$$

(d) Memory storage function of the node $X_{i}$ at time $t$ which is denoted by $s_{i}(t)$.

Now let us give a system of equations among these four types of functions:

I. State equation $(i=1,2, \cdots, N)$

$$
X_{i j}(t+\tau)=A_{i j}\left[X_{i j}(t), t ; e_{i}(t), s_{i}(t)\right] .
$$

II. Output equation $(i, j=1,2, \cdots, N)$

$$
O_{i j}(t+\tau)=B_{i j}\left[O_{i j}(t), t ; e_{i}(t), s_{i}(t)\right] .
$$

III. Input equation $(i, k=1,2, \cdots, N)$

$$
e_{k, i}(t+\tau)=C_{k, i}\left[e_{k, i}(t), t ; s_{i}(t)\right] \text {. }
$$

IV. Memory storage equation $(i=1,2, \cdots, N)$

$$
s_{i}(t+\tau)=D_{i}\left[s_{i}(t), t ; X_{i}(t), e_{i}(t)\right] .
$$

Here each of the functions $A_{i j}, B_{i j}, C_{k i}$ and $D_{i}$ may be deterministic, stochastic or even fuzzy in the sense discussed in our paper [12]. In any case we are concerned with network system of automata arranged in some parallel way.

The main aspects of the problems with which we should be concerned can be classified into the following three aspects:

(a) Eizon aspect

Whether or not and how long the whole network system can continue to subsist with keeping its safety? The meaning of the key notion eizon is explained in a series of our papers [3], [4], [9], [12].

(b) Control aspect

Under a certain set of conditions which assign the functional forms in (5.04), (5.07), how far can we increase the efficiency of the network system by suitable choice of parameters in them?

(c) Creation aspect

How can we set up another type of network system under the conditions that the functional forms in (5.04) can be chosen under our command? 
The reason why we are referring to the feasibility of the network system is due to the fact that there is naturally a certain domain of existence for each information system which is defined by a set of threshold values or by threshold domain and which should be imposed on information architectures, as something similar to strength restrictions to be imposed on material architectures in view of material dynamics. Throughout the main aspects (a), (b) and (c), the most essential features of our solutions will be concerned with some answers to the following questions: (i) to show whether or not there do exist some stable equilibrium states; (ii) to show whether or not the system can either keep its level of eternal stability or can move to another level of stability after passing through transient states; (iii) to show whether or not structural stability does hold; (iv) to discuss whether or not it is possible to create a new type of stable equilibrium.

In this connection we should remark that the existence of threshold domains in our social network approach can be satisfied by the assumption that dynamical behaviours of our system in terms of the functions $\left\{X_{i}(t)\right\}(i=1,2, \cdots, N)$ are defined by a system of non-linear equations of certain types. This is quite an essential property of our system of equations because there will be then the possibility of pulling-off phenomena in the sense of Wiener [21] which yields to our system of equations the possibility of self-organizing process of the social network system as the Wiener theory [21] may suggest.

We are not going here to enter into detailed discussions on the various problems which we have proposed to present just now. These must be discussed in a systematic way in another occasion. Nevertheless we may and we shall enunciate some of the important implications of our social network system approach to sociological and historical sciences.

$\left(1^{\circ}\right)$ The stimulus-response relationship among the $N$ nodes should be better understood and interpretated as the adaptation relationship implying assimilation and accomodation processes.

$\left(2^{\circ}\right)$ The three feasibility aspects of existence, which we coin as (i) eizon, (ii) control and (iii) creation, should be understood and interpretated also from the framework of equilibrium and equilibration in the sense of J. Piaget [17], [18].

$\left(3^{\circ}\right)$ Each of the five civilization architectures called (I) enchantment, (II) mythology, (III) religion, (IV) technology, and (V) science respectively can be regarded as having its specific social communication scheme upon which its foundation and its development are based. It will be interesting to set up a mathematical model of social information network system which can describe its specific social communication scheme. The comparison among these mathematical models each of which corresponds to each of these five civilization architectures will contribute to clarify the mutual relationship and the conditions for transitions among these five civilization architectures. In this connection there are two essential assertions $\left(1^{\circ}\right)$ and $\left(2^{\circ}\right)$ which we want to emphasise here. (1 $\left.1^{\circ}\right)$ Our first essential assertion is that, in spite of various possible ways for comparison of these five civilization architectures, a formulation of information network system associated with each civilization architecture may be one of the most simple and the most fruitful ap- 
proaches. $\left(2^{\circ}\right)$ Our second essential assertion is that we recommend a mathematical formulation of the specific type given in (5.01) which is general enough for us to every civilization architecture and simple enough to concentrate to its most essential aspects without entering into its minor details.

It should be pointed out that there is one specific node which represents the nature itself. Let us consider for instance the relationship between (I) enchantment and (IV) technology. It is true that there are several common features between (I) enchantment and (IV) technology, which derives from a certain set of the common structures between their respective information network system so far as they are concerned with control aspects of evaluation schemes. Nevertheless there is one definite difference between (I) and (IV) which comes from the difference of channel connections of all nodes with one particular node called the nature. That is to say, the difference between (I) and (IV) is in fact mainly due to the quality of information obtained from the nature, to the communication procedures of carrying the information thus being obtained from the nature, and to the feedback procedures by which learning from the nature will be established. The comparison between (III) religion and (V) science will show that, in spite of their common features, there exists also one sharp difference between them which comes again from the difference analogous to the difference between (I) enchantment and (IV) technology, that is, the difference of the information network system regarding the nature.

In this connection we should remark that we are far from the viewpoints of behaviourism based upon stimulus-response relationship. It is the opinion of the present author that the famous work of Mead [15] should be better understood from our present approach based upon neural equations and informative logics than from what he himself relied upon as social behaviourist.

\section{References}

[ 1 ] Caianiello, E.R.: Outline of a thought-processes and thinking machines, Journ. Theoret. Biology 2 (1961), 204-235.

[ 2 ] Codp, E.F.: Cellular automata, Academic Press, New York and London, 1968.

[ 3 ] Kitagawa, T.: The logic of information science (In Japanese), Tokyo, Kodansha, Caontemporary Series, Vol. 200, 1969.

[ 4 ] KITAGAWA, T.: Three coordinate systems for information science approaches, Information Sciences, 5 (1973), 157-169.

[ 5 ] Kitagawa, T.: Cell space approaches in biomathematics, Math. Biosciences, 17 (1973).

[6] KITAGAwA, T.: Dynamical systems and operators associated with a single neuronic equation, Math. Biosciences 18 (1973), 191-244.

[ 7 ] Kitagawa, T.: Biorobots for simulation studies of learning and intelligent control, learning systems and intelligent robots, Ed. K. S. Fu and J. T. Tou, Plenum Publishing Corp., (1974), $33-46$.

[ 8 ] KITAGAWA, T.: Brainware concept in intelligent and integrated system of information, FID/RI Committee Meeting "Information science, its scope, objects and problems", Moscow, April 24-26, (1974) (to be published).

[9] KITAGAwA, T.: The logics of information sciences, and its implication for control processes in large systems, Second Formator Symposium on Mathematical Methods for the Analysis 
of Large Scale Systems, Proceedings of a Symposium held in Prague, June 18-21, 1974, Academia Prague 13-31.

[10] Kitagawa, T.: Historical images of human civilizations A prolegomena to informative conception of history (In Japanese), Series of Social Information Science, Vol. 17, Views for information oriented culture, No. 1, Gakkuken (1974).

[11] KitAGAWA, T.: Dynamical behaviours associated with a system of $N$ neuronic equations with time lag, (1)-(3), Research Institute of Fundamental Information Science, Kyushu Univ., Research Report No. 46, No. 49 and No. 54 (1975). (to appear)

[12] Kitagawa, T.: Fuzziness in informative logics, Fuzzy sets and their applications to cognitive and decision processes (ed. by Zadeh. A, Tanaka, K \& others), Acad. Press, 1975, 97-124.

[13] KITAgAwa, T.: Dynamics of reverberation cycles and its implications to linguistics, Research Institute of Fundamental Information Science, Kyushu Univ., Research Report No. 56 (1975). (to appear)

[14] Lenneberg, E.H.: Biological foundations of language, John Wiley \& Sons, New York/ London/Sydney, 1967.

[15] Mead, G.: Mind, Self, and Society: from the Standpoint of a Social Behaviourist, University of Chicago Press, 1934.

[16] Minsky, M. and Papert, S.: Perceptrons-An Introduction to Computational Geometry, M. I. T. Press, 1969.

[17] Piaget, J.: The Psychology of Intelligence, (Translated from the French by Piercy, M. and Berklyne, D.E.), Poutledge \& Kegan Paul Ltd., London, 1950.

[18] Piaget, J.: Genetic Epistemology (Translated by Duckwork, E.) Columbia Univ. Press, New York, 1970.

[19] Thом, R.: Stabiilté structurelle et morphogénèse W.A. Benjamin, New York 1972.

[20] Tном, R.: Topologie et Signification, L'Age de la Science, No. 4, Dunod, Paris, (1968), $1-24$.

[21] WiEner, N.: Nonlinear problems in random theory, John Wiley \& Sons, MIT Press (1958). 